\title{
On Space, Time, and Language: For the Next Century, Timing Is (Almost) Everything
}

\author{
Lee Osterhout
}

University of Washington

With the advent of neuroimaging tools has come a surpassing concern for space and a corresponding disregard for time. The next century will see a newfound appreciation of the fact that, with respect to language, time is always of the essence.

Neuroimaging tools such as functional magnetic resonance imaging (fMRI) and positron emission tomography (PET), when tweaked to measure changes in blood flow over time, can be used to identify brain regions that are active during a specific cognitive task, relative to some baseline task. These tools provide (by historical standards) an impressive degree of spatial resolution and have proved helpful in revealing the neurobiology of certain cognitive functions (e.g., visual perception; Tootell et al., 1998). Less success has been achieved in elucidating the neurobiology of language.

Why is this? From the perspective of the person trying to comprehend language, language is a continuous flow of information distributed over time. Somehow, the listener translates this stream of information into discrete and rapidly sequenced units of phonemes, meaning, and structure, and does so in real time. The primary question facing us is, of course, how people accomplish this. My contention is that in addressing this question, where in the brain activity is found is intimately related to when this activity is occurring, and both the where and the when of the activity must be considered to fully appreciate what is happening at that particular time and space.

Let me illustrate. Numerous imaging studies have reported activation in the inferior frontal lobe of the left hemisphere (in or near Broca's area) when words or sentences are presented as stimuli. This activity has been at-

Preparation of this paper was supported by research Grant R01DC01947 from the National Institute on Deafness and Other Communication Disorders, National Institutes of Health.

Address correspondence and reprint requests to Lee Osterhout, Department of Psychology, Box 351525, University of Washington, Seattle, WA 98195. E-mail: losterho@u. washington.edu. 
tributed to (among other things) phonological processing, semantic processing, syntactic processing, working memory, and willed action. I propose that the critical constraint needed to definitively answer the what question is time. This follows from the observation that neural manifestations of these (or relevant correlated) processes are temporally distinct and transient events, as revealed by the excellent temporal resolution provided by eventrelated potentials (ERPs) and magnetoencephalography (MEG; cf. Osterhout, McLaughlin, \& Bersick, 1997). The problem is that the hemodynamic response to an event is delayed in onset and evolves over 10 to $12 \mathrm{~s}$, which is an order of magnitude or two slower than the (phonological, syntactic, semantic, etc.) processes of interest. In other words, in imaging experiments all of these processes are represented in the time dimension by a single data point. This is as true for event-related fMRI designs (e.g., Burock et al., 1998 ) as it is for standard blocked-trial designs. By contrast, ERPs and MEG provide a sampling of activity every few milliseconds, thereby allowing discrimination between closely sequenced transient events.

The challenge facing us in the upcoming decades is to overcome this problem in imaging time. One approach already in use combines neuroimaging data with ERP or MEG data. Assuming that the neural generators of the ERP activity can be modeled as a small number of discrete dipole sources, the location of the ERP generators can be compared to the activated regions revealed by imaging (see, e.g., Heinze et al., 1994). Moreover, ERP effects occurring at different times can be mapped onto different activations within a single map of activation (Snyder et al., 1995). However, to help identify what is happening at a particular place, this approach requires knowledge about the functional significance of the corresponding ERP/MEG effect. The future must also bring with it a means for dealing with the (numerous) ERP sources that cannot be modeled as simple dipoles and, critically, a much better understanding of how rapid changes in electromagnetic activity relate to much slower changes in blood flow.

Another approach dispenses with imaging altogether. Recent evidence indicates that transient $(\sim 50 \mathrm{msec})$ synchronization of neuronal discharges acts as an integrative mechanism to bind widely distributed neurons into a coherent ensemble (Rodriguez et al., 1999). Different ensembles of synchronized neurons are claimed to represent different cognitive tasks or states. This transient activity, occurring in the gamma frequency range $(30-80 \mathrm{~Hz})$, is thought to reflect Hebbian associative learning (Pulvermüller, 1996). Preliminary research indicates that some aspects of word processing can be characterized in these terms (Pulvermüller, 1996). The next decades will reveal whether (and which) specific language processes are uniquely characterized spatially and temporally by such transient synchronizations (and, by inference, by associative processes).

In the next century, the prospects for learning more about how the brain enables language depend mostly on the successful introduction of the time 
dimension into the heretofore three-dimensional spatial maps of activation. Otherwise, the cognitive neuroscience of the future might quickly become a relic of the past.

\section{REFERENCES}

Burock, M. A., Buckner, R. L., Woldorff, M. G., Rosen, B. R., \& Dale, A. 1998. Randomized event-related experimental designs allow for extremely rapid presentation rates using functional MRI. Neuroreport, 9, 3735-3739.

Heinze, H. J., Mangun, G. R., Buchert, W., Hinrichs, M., Scholtz, M., Munte, T. F., Gos, A., Scherg, M., Johannes, S., Hundeshagen, H., Gazzaniga, M., \& Hillyard, S. A. 1994. Combined spatial and temporal imaging of brain activity during visual selective attention in humans. Nature, 372, 543-546.

Osterhout, L., McLaughlin, J., \& Bersick, M. 1997. Event-related potentials and human language. Trends in Cognitive Sciences, 1, 203-209.

Pulvermüller, F. 1996. Hebb's concept of cell assemblies and the psychophysiology of word processing. Psychophysiology, 33, 317-333.

Rodriguez, E., George, N., Lachaux, J., Martinerie, J., Renault, B., \& Varela, F. J. 1999. Perception's shadow: Long-distance synchronization of human brain activity. Nature, 397, 430-433.

Snyder, A. Z., Abdullaev, Y. G., Posner, M. I., \& Raichle, M. E. 1995. Scalp electrical potentials reflect regional cerebral blood flow responses during processing of written words. Proceedings of the National Academy of Sciences, 92, 1689-1693.

Tootell, R. B. H., Hadjikhani, N. K., Mendola, J. D., Marrett, S., \& Dale, A. M. 1998. From retinotopy to recognition: fMRI in human visual cortex. Trends in Cognitive Sciences, 2, 174-183. 